\title{
Reflectarray Antenna with Reduced Crosspolar Radiation Pattern
}

\author{
Daniel R. Prado , Manuel Arrebola , Marcos R. Pino , Fernando Las-Heras \\ Rafael Florencio, Rafael R. Boix and José A. Encinar
}

\begin{abstract}
A method for the optimization of the crosspolar component of dual-polarized reflectarrays using the Method of Moments (MoM) is described. It is based on the LevenbergMarquardt Algorithm (LMA) and it exploits several optimization techniques to accelerate the computations in order to be able to use MoM within acceptable computing times. Since MoM is used to analyze the reflectarray unit cell, it accurately accounts for the crosspolar components of the radiated far field, which can then be optimized in order to reduce its value while preserving the copolar shape. A test case is provided to validate the developed algorithm in which a Local Multipoint Distribution Service (LMDS) pattern is optimized.
\end{abstract}

Index Terms-Crosspolar optimization, Local Multipoint Distribution System (LMDS), reflectarray, Levenberg-Marquardt.

\section{INTRODUCTION}

Until recently, reflectarray designs were performed based on a prescribed phase distribution which provided the desired pattern, being a conveniently focused pencil beam or a shaped pattern [1]. The dimensions of the reflectarray elements were adjusted in such a way that the desired phase distribution was accomplished, providing the desired copolar pattern. However, there was no control over the crosspolar far field during the synthesis and design processes.

One of the first attempts to reduce the crosspolar radiation pattern was presented in [2] and was based on properly adding a constant to the phase distribution in order to impose a $180^{\circ}$ phase shift and amplitude null in the cross-coefficients that contribute more to the crosspolar pattern. However, this approach is not very flexible, since the reduction in the crosspolar level is performed after the synthesis. In [3] a direct optimization of the crosspolar component of singlepolarized reflectarrays using directly the Method of Moments (MoM) was presented, resulting in a very slow algorithm. More recently, new techniques have appeared for the crosspolar optimization of dual-polarized reflectarrays. In [4], after a Phase-Only Synthesis (POS), the crosspolar pattern was suppresed and the reflection coefficients obtained using back propagation techniques. Then, the element of the reflectarray was appropriately modified using geometrical transformations to match the required coefficients. In [5] a direct optimization technique was developed based on a gradient minimax and the use of a database in order to avoid the direct use of MoM during the optimization process. Another approach is provided in [6] where an efficient generalization of the Intersection Approach algorithm is employed to synthesize the complex reflection coefficients which provide the desired radiation patterns with both copolar and crosspolar specifications.

In this contribution, a technique for the crosspolar optimization of dual-polarized planar reflectarrays is presented. It is based on the Levenberg-Marquardt Algorithm (LMA) and uses MoM in order to analyze the unit cell. Several optimization techniques are used that allow to use MoM directly in the optimization algorithm within acceptable computing times. A test case consisting on a Local Multipoint Distribution System (LMDS) is presented in which, after a POS, the crosspolar radiation pattern is optimized, reducing its maximum value while preserving the desired copolar shape.

\section{REFLECTARRAY ANALYSIS}

A sketch of the reflectarray geometry under consideration is shown in Figure 1. It consists of a feed which illuminates the reflectarray with an incident field $\vec{E}_{\text {inc }}$, which can be expressed as

$$
\vec{E}_{\text {inc }}^{X / Y}(x, y)=E_{\text {inc }, x}^{X / Y}(x, y) \hat{x}+E_{\text {inc }, y}^{X / Y}(x, y) \hat{y},
$$

where the superscripts indicate the polarization of the feed and the subscript the field component with regard to the reflectarray coordinate system. Similarly, the reflected field can be written as

$$
\vec{E}_{\mathrm{ref}}^{X / Y}(x, y)=E_{\mathrm{ref}, x}^{X / Y}(x, y) \hat{x}+E_{\mathrm{ref}, y}^{X / Y}(x, y) \hat{y},
$$

and is related to the incident field through the expression [1]

$$
\vec{E}_{\text {ref }}^{X / Y}\left(x_{m}, y_{m}\right)=\mathbf{R}^{m n} \cdot \vec{E}_{\text {inc }}^{X / Y}\left(x_{m}, y_{m}\right)
$$

where

$$
\mathbf{R}^{m n}=\left(\begin{array}{cc}
\rho_{x x}^{m n} & \rho_{x y}^{m n} \\
\rho_{y x}^{m n} & \rho_{y y}^{m n}
\end{array}\right),
$$

and $\left(x_{m}, y_{n}\right)$ are the coordinates of the $(m, n)$ th element. Matrix $\mathbf{R}^{m n}$ in (4) is known as the matrix of reflection 


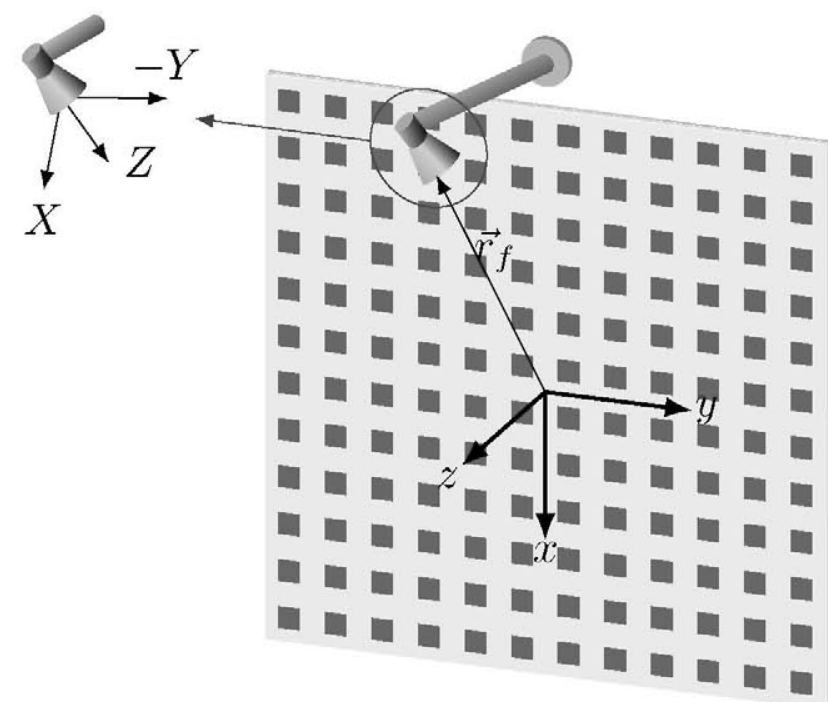

Figure 1. Sketch of the reflectarray geometry.

coefficients, which are complex and fully characterize the behavior of the unit cell. They are typically computed by using a full wave technique, such as MoM, Finite Element Method (FEM) or Finite Difference Time Domain (FDTD) [1].

Once the tangential field in (3) is obtained, the radiation patterns can be efficiently obtained by means of the FFT applying the first [7] or second [1] principle of equivalence. For dual-polarized reflectarrays, four far fields are generated, copolar and crosspolar for two polarizations, which will have to be taken into account in the optimization process.

\section{CROSSPOLAR OPTIMIZATION BASED ON LMA}

The LMA has been chosen to directly optimize the reflectarray geometry (i.e. the elements dimensions) using MoM, assuming local periodicity and taking into account the real angle of incidence from the feed [1]. It consist of several building blocks which will have to be conveniently optimized in order to reduce computing times as much as possible. First, the employed cost function to be minimized by the algorithm is the same used in [8] but modified to incorporate the four radiation patterns. The analysis of the reflectarray elements necessary to obtain the far fields is parallelized by means of OpenMP [9] in order to accelerate the MoM computations, by processing one element per available thread.

The most time consuming operation of the algorithm is the computation of the Jacobian matrix. It is a $4 T \times m P$ matrix, where $T$ is the number of samples of each radiation pattern, $P$ the number of reflectarray elements that are being optimized and $m$ the number of optimization variables per element of the reflectarray. When performing a POS, there is an easy analytical expression relating the optimization variables and the cost function, and thus the derivatives can be easily obtained analytically. However, when optimizing directly the geometry, this is not possible, and finite differences must be employed. Since the Jacobian matrix evaluation calls the cost function routine, which employs MoM, it can be extremely slow.
However, since each column of the Jacobian only depends on the modification of one element of the reflectarray, there is no need to fully analyze the reflectarray, but only the element in question. This way, each column of the Jacobian only requires one call to the MoM routine, since the analysis is based on local periodicity. (Please note that if the full-wave analysis was carried out for the entire reflectarray this enhancement would not be possible and the whole optimization process would take huge amounts of time.) Furthermore, the evaluation of this matrix can be also parallelized by computing one column per available thread, further saving computing times.

In order to form the normal equations, some matrix and matrix-vector multiplications have to be performed. These operations can be carried out by an optimized BLAS implementation such as OpenBLAS [10] or Intel MKL [11], which are implemented with low-level hardware instructions and are fully parallelized. Also, in the matrix multiplication, the resulting matrix is symmetric and only the upper or lower part of it needs to be computed, further saving time.

Finally, before updating the solution, a system equation needs to be solved. Due to the nature of the problem, the matrix of the equation system is at least positive semidefinite and a Cholesky factorization based solver can be used, which is the fastest exact solver for this type of problems. The one provided in the Intel MKL library is employed [11].

\section{VALIDATION}

\section{A. Antenna specifications}

A planar rectangular reflectarray has been chosen consisting of 900 elements $(30 \times 30)$ and illuminated by a feed horn modeled as a $\cos ^{q} \theta$ function with a $q$-factor of 37, which produces an illumination taper of $-19.7 \mathrm{~dB}$ at the edges of the reflectarray. The phase center of the feed horn is placed at $\vec{r}_{f}=(-94,0,214) \mathrm{mm}$. with regard to the center of the reflectarray (see Figure 1). The working frequency is $25.5 \mathrm{GHz}$. and the periodicity of the cells is $5.84 \mathrm{~mm} \times 5.84 \mathrm{~mm}$, which is approximately half a wavelength. The chosen unit cell for the design is the one shown in [12, Figure 6]. Arlon CuClad 233LX, with $\varepsilon=2.33, \tan \delta=0.0013$ and thickness $0.787 \mathrm{~mm}$ has been chosen for the two layers of the substrate.

\section{B. Starting point}

The starting point for the crosspolar optimization is the POS from [13]. From the phase distribution and using a zero-finding routine, the layout of the reflectarray is found [1]. Then, the algorithm will directly optimize the geometry analyzing the unit cell with MoM. The figure of merit to evaluate the improvement is the difference between the maximum value of the copolar and the crosspolar components, and will be denoted as CP-XP. For the case at hand, the initial CP-XP is $24.89 \mathrm{~dB}$ and $24.88 \mathrm{~dB}$ for the $\mathrm{X}$ and $\mathrm{Y}$ polarizations, respectively. Figure 2 shows the radiation pattern of the starting point for the $X$ polarization, being the one for $\mathrm{Y}$ polarization very similar. 


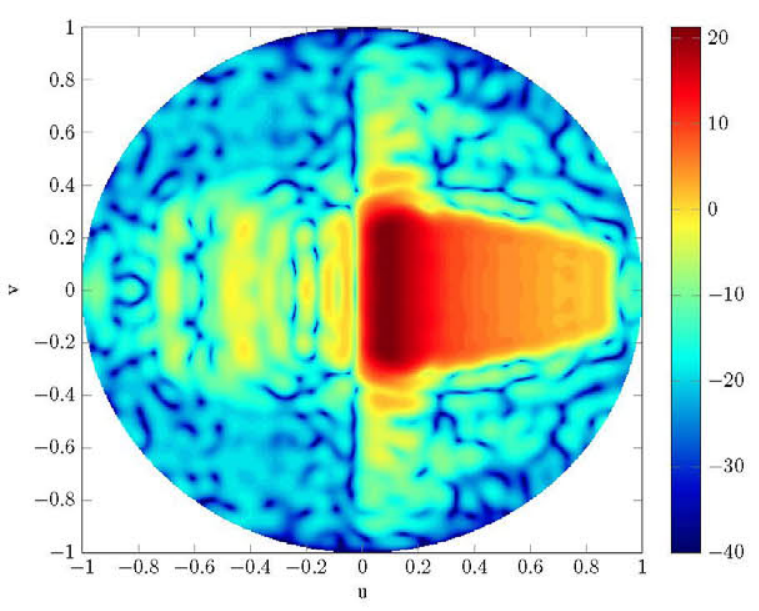

(a)

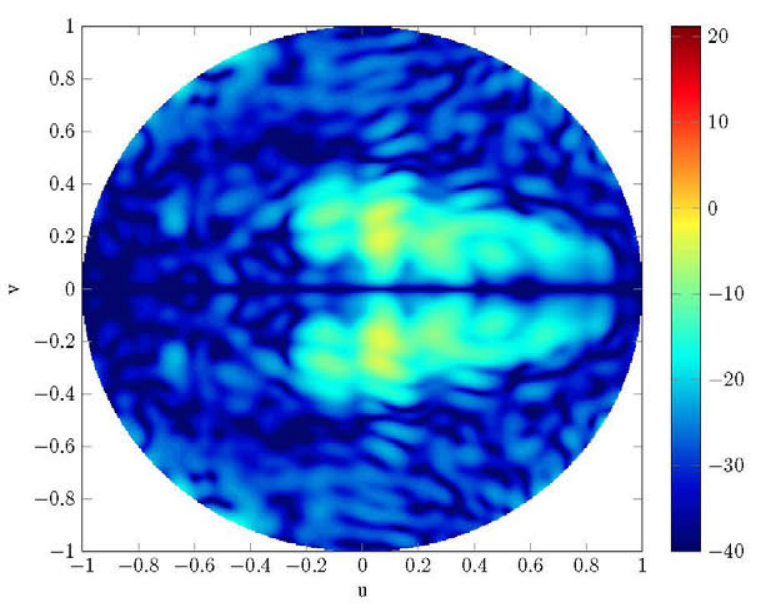

(b)

Figure 2. Radiation patterns in directivity $(\mathrm{dB})$ at the starting point for $\mathrm{X}$ polarization. The CP-XP value is $24.89 \mathrm{~dB}$. (a) Copolar. (b) Crosspolar.

\section{Results}

The algorithm was tested in two different computers to better appreciate its scalable capabilities. The first computer was an Intel Core i3-2100, with two physical and two virtual CPU working at $3.10 \mathrm{GHz}$, where the algorithm took around 360 seconds per iteration and 4.16 days to complete 999 iterations. The other computer was an Intel Xeon CPU E5-2650 v2 with 16 physical and 16 virtual CPU working at $2.6 \mathrm{GHz}$. In this case, the algorithm took around 53 seconds per iteration and 14.9 hours to complete 999 iterations.

The results after the optimization are shown in Figures 3, 4 and 5. The directivity of the antenna remains the same and the copolar patterns mostly remain within the templates, as it can be seen in Figure 3. However, the crosspolar level is reduced. Now, CP-XP is $29.48 \mathrm{~dB}$ for X-pol. and $28.82 \mathrm{~dB}$ for Y-pol, which supposes an improvement of the crosspolar component of $4.59 \mathrm{~dB}$ and $3.94 \mathrm{~dB}$ for $\mathrm{X}$ and $\mathrm{Y}$ polarizations, respectively. The reduction in the crosspolar component causes that the level of crosspolarization is more evenly distributed in the whole visible region and the increment of the secondary

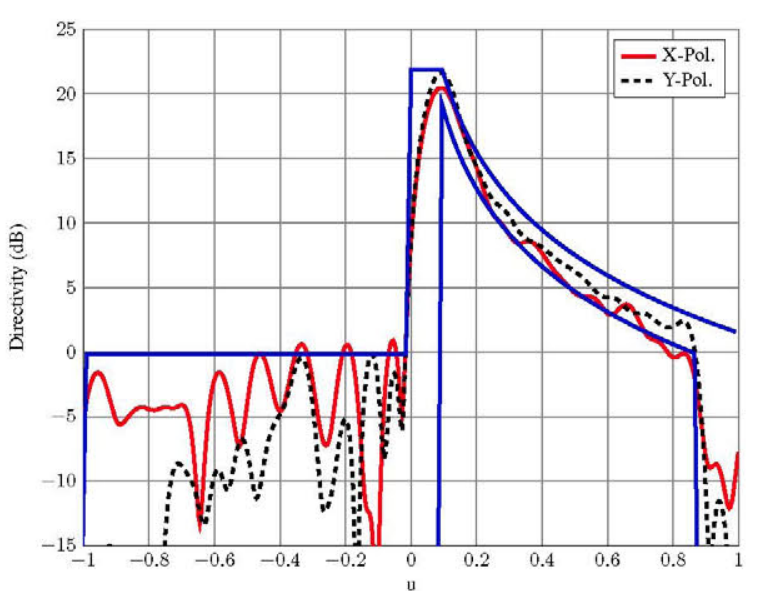

(a)

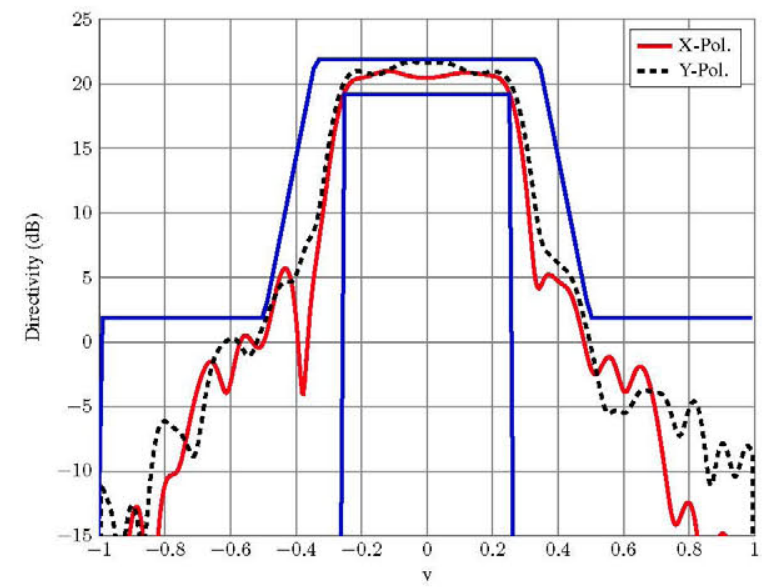

(b)

Figure 3. Main cuts of the optimized radiation patterns. (a) Cut along $u$ (b) Cut along v.

lobes in the copolar pattern. The copolar radiation pattern for $\mathrm{X}$-polarization is slightly affected by the optimization process (compare Figures 2 and 4), although it almost complies with the specifications. The cost function could include a weighting function in order balance the crosspolar reduction with the copolar deterioration, in those cases in which the copolar pattern is more affected. Finally, Figure 5 shows the crosspolar cuts before and after the optimization, where the crosspolar patterns present their maximum value.

\section{CONCLUSION}

The Levenberg-Marquardt algorithm has been adapted to optimize the crosspolar component of reflectarray antennas using MoM in the computations. Because the MoM routine is slow, the algorithm has been fully optimized in order to take advantage of the resources available. In particular, all building blocks of the algorithm have been conveniently parallelized and optimized in order to reduce the number of MoM calls. An optimization has been carried out in two different computers in order to assess the scalability of the proposed technique. An LMDS radiation pattern has been chosen as a test case in 


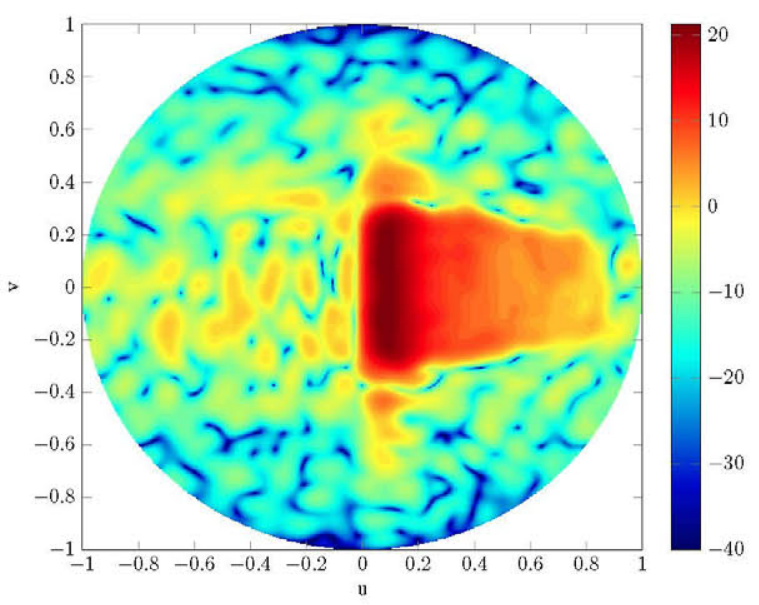

(a)

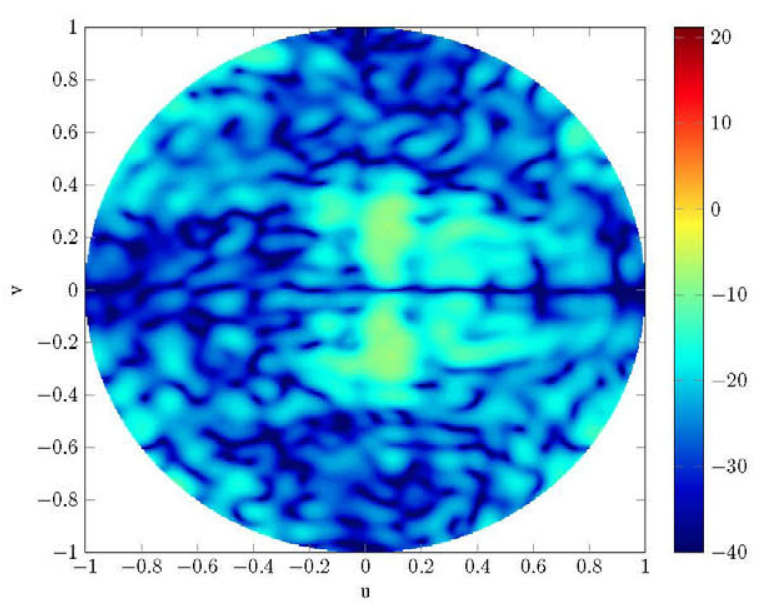

(b)

Figure 4. Optimized radiation patterns in directivity $(\mathrm{dB})$ for $\mathrm{X}$ polarization. The CP-XP value is $29.48 \mathrm{~dB}$. (a) Copolar. (b) Crosspolar.

which the crosspolar far field has been reduced $4.59 \mathrm{~dB}$ and $3.94 \mathrm{~dB}$ for the $\mathrm{X}$ and $\mathrm{Y}$ polarizations, respectively.

\section{REFERENCES}

[1] J. Huang and J. A. Encinar, Reflectarray Antennas. John Wiley \& Sons, 2008.

[2] J. A. Encinar and M. Arrebola, "Reduction of cross-polarization in contoured beam reflectarrays using a three-layer configuration," in IEEE Antennas and Propagation Society International Symposium, Honolulu, Hawaii, USA, Jun. 9-15, 2007, pp. 5303-5306.

[3] O. M. Bucci, A. Capozzoli, G. D'Elia, and S. Musto, "A new approach to the power pattern synthesis of reflectarrays," in Proc. URSI International Symposium on Electromagnetic Theory (EMTS'04), Pisa, Italy, May 23 27, 2004, pp. 1053-1055.

[4] H. Legay, D. Bresciani, E. Labiole, R. Chiniard, and R. Gillard, "A multi facets composite panel reflectarray antenna for a space contoured beam antenna in ku band," Progr. Electromagn. Res. B, vol. 54, pp. 1-26, Aug. 2013.

[5] M. Zhou, S. B. Sørensen, O. S. Kim, E. Jørgensen, P. Meincke, and O. Breinbjerg, "Direct optimization of printed reflectarrays for contoured beam satellite antenna applications," IEEE Trans. Antennas Propag., vol. 61, no. 4, pp. 1995-2004, Apr. 2013.

[6] D. R. Prado, M. Arrebola, M. R. Pino, and F. Las-Heras, "Complex reflection coefficient synthesis applied to dual-polarized reflectarrays with cross-polar requirements," IEEE Trans. Antennas Propag., vol. 63 , no. 9, pp. 3897-3907, Sep. 2015.

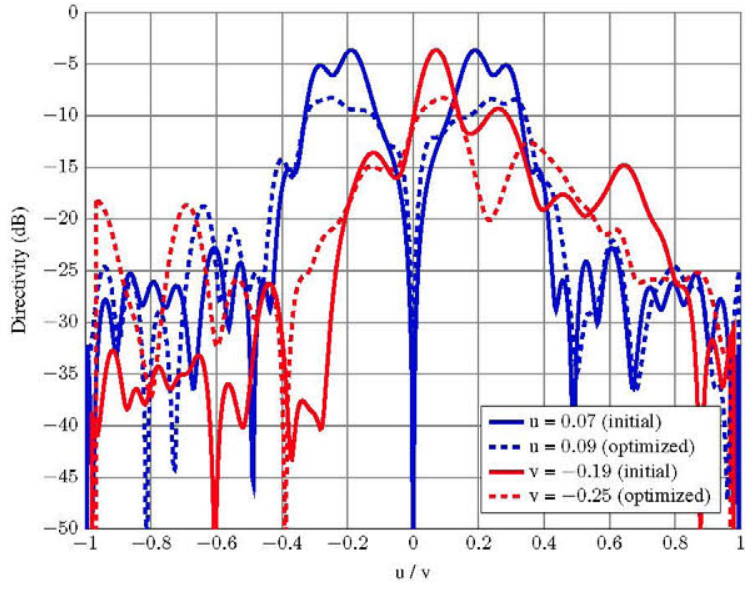

(a)

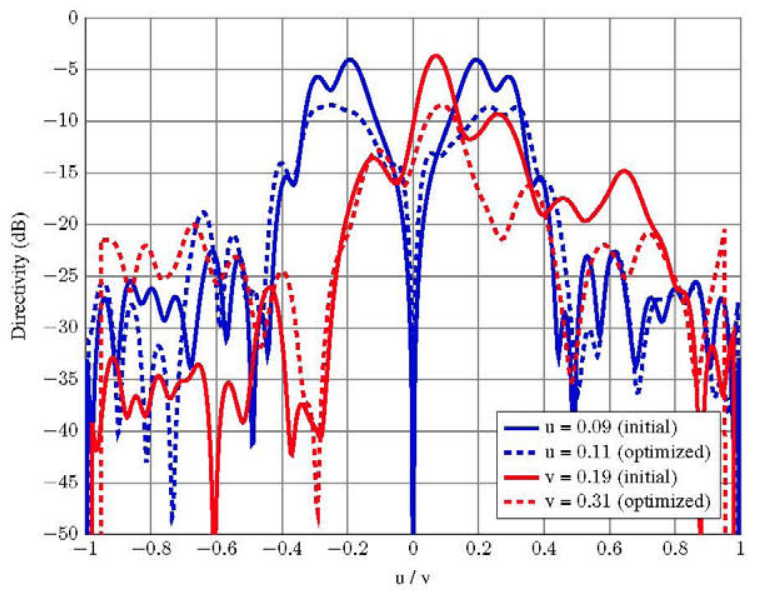

(b)

Figure 5. Cuts along $\mathrm{u}$ and $\mathrm{v}$ of the crosspolar far field before and after the optimization. The cuts are taken at the point where the crosspolar level is maximum for each pattern. (a) X polarization. (b) Y polarization.

[7] M. Zhou, S. B. Sørensen, E. Jørgensen, P. Meincke, O. S. Kim, and $\mathrm{O}$. Breinbjerg, "An accurate technique for calculation of radiation from printed reflectarrays," IEEE Antennas Wireless Propag. Lett., vol. 10, pp. 1081-1084, 2011.

[8] J. Álvarez, M. Arrebola, R. G. Ayestarán, and F. Las-Heras, "Systematic framework for reflectarray synthesis based on phase optimization," Int. J. Antennas Propag., vol. 2012, pp. 1-9, Jun. 2012.

[9] M. Sato, "OpenMP: parallel programming API for shared memory multiprocessors and on-chip multiprocessors," in 15th International Symposium on System Synthesis, Kyoto, Japan, Oct. 2-4, 2002, pp. 109 111.

[10] Z. Xianyi, W. Qian, and Z. Yunquan, "Model-driven level 3 BLAS performance optimization on loongson 3a processor," in IEEE 18th International Conference on Parallel and Distributed Systems (ICPADS), Singapore, Dec. 17-19, 2012, pp. 684-691.

[11] Intel ${ }^{\circledR}$ Math Kernel Library Reference Manual, Intel Corporation, Aug. 2008.

[12] R. Florencio, J. A. Encinar, R. R. Boix, V. Losada, and G. Toso, "Reflectarray antennas for dual polarization and broadband telecom satellite applications," IEEE Trans. Antennas Propag., vol. 63, no. 4, pp. 1234-1246, Apr. 2015.

[13] M. Arrebola, J. A. Encinar, and M. Barba, "Multifed printed reflectarray with three simultaneous shaped beams for LMDS central station antenna," IEEE Trans. Antennas Propag., vol. 56, no. 6, pp. 1518-1527, Jun. 2008. 